\title{
2019 CSHP National Awards Program Winners Programme national des prix 2019 de la SCPH : lauréats et lauréates
}

The winner of the Distinguished Service Award (sponsored by Pharmascience Inc.) is Moira Wilson (Saint John, NB).

The winner of the Isabel E. Stauffer Meritorious Service Award (sponsored by Fresenius Kabi Canada Ltd.) is Diane Brideau-Laughlin (Moncton, NB).
The winner of the New Hospital Pharmacy Practitioner Award (sponsored by SteriMax Inc.) is Laura V Minard (Halifax, NS).

The winner of the Hospital Pharmacy Student Award (co-sponsored by the Canadian Society of Hospital Pharmacists [CSHP] and the Canadian Association of Pharmacy Students and Interns [CAPSI]) is Sydney Evans (Riverview, NB).
Patient Care Enhancement Award Sponsored by Teva Canada Limited Benzodiazepine and Sedative-Hypnotic Drug Use in Nova Scotia Hospitals: A Point Prevalence Survey (completed at Nova Scotia Health Authority)

Heather L Neville, Mia Losier, Jennifer Pitman, Melissa Gehrig, Jennifer Isenor, Laura V Minard, Ellen Penny, Susan K Bowles

Pharmacotherapy Best Practices Award Sponsored by Pfizer Canada ULC

Voriconazole Prophylaxis in Leukemic Patients: A Retrospective Single Centre Study (completed at Sunnybrook Health Sciences Centre) Vivian Bui, Sandra A N Walker, Marion Elligsen

\section{Safe Medication Practices Award}

Sponsored by HealthPRO Procurement Services Inc. Retrospective, Multicentre Matched Cohort Study Comparing Safety and Efficacy Outcomes of Intermittent Infusion and Continuous Infusion Vancomycin (completed at Sunnybrook Health Sciences Centre) Nathan $\mathrm{HMa}$

Teaching, Learning and Education Award Sponsored by Pfizer Canada ULC

Development of a Vancomycin Competency-Based Training and Assessment Program (VC-TAP) (completed at Saskatchewan Health Authority, Saskatoon Area) Meagan Rieger, Barb Evans, Justin Kosar

The award-winning abstracts are published exactly as submitted by the authors and have not undergone any copyediting by the Canadian Journal of Hospital Pharmacy. Le Journal canadien de la pharmacie hospitalière n’a pas soumis les résumés primés à une révision linguistique et les publie ici tels que remis par les auteurs.

\section{Benzodiazepine and Sedative-Hypnotic Drug Use in Nova Scotia Hospitals: A Point Prevalence Survey Patient Care Enhancement Award, sponsored by Teva Canada Limited \\ Neville $H L^{1}$, Losier $M^{1,2}$, Pitman ${ }^{1,2}$, Gehrig $M^{1}$, Isenor $J E^{2}$, Minard $L V^{1}$, Penny $E^{1}$, Bowles $S K^{1,2}$ \\ ${ }^{1}$ Department of Pharmacy, Nova Scotia Health Authority, Nova Scotia ${ }^{2}$ College of Pharmacy, Dalhousie University, Halifax, NS}

Background: Benzodiazepines and sedative-hypnotic drugs (BZD/ SHDs), such as zopiclone and the antidepressant trazodone, pose a number of risks such as falls, fractures, and confusion, especially in older adults. Use of these drugs is poorly understood in the acute care setting. Objectives: To determine the point prevalence and characterize use of BZD/SHDs in Nova Scotia hospitals with $\geq 30$ acute care beds.

Methods: A point prevalence survey (PPS) was conducted by reviewing the health records of adults admitted to hospital between May and August 2016. The number of patients who received a BZD/SHD within the 24 hours prior to the start of the survey was divided by the total number of patients admitted to each ward to determine prevalence. Exclusions were patients $<18$ years of age, drugs given by the intravenous route, and patients in long term care, mental health, addiction treatment, or critical care. Results were summarized descriptively.
Results: All 16 eligible hospitals participated in the PPS. The overall prevalence of BZD/SHD use was 34.6\% (487/1409) and ranged from $15.6-56.3 \%$. The average age of patients who received a BZD/SHD was 70.3 years, $30.8 \%$ of patients were $\geq 80$ years of age, and $54.6 \%$ of patients were female. The most commonly used drugs included zopiclone $(32.0 \%)$, lorazepam $(21.9 \%)$, trazodone $(21.9 \%)$ and clonazepam (11.3\%). Top indications for use were bedtime/daytime sedation $(60.0 \%)$ and anxiety (12.5\%). In $17 \%$ of cases the indication could not be determined. Over half $(55.7 \%)$ of the medications had been initiated at home and continued in hospital, the remainder were started in hospital (37.6\%), or unknown (6.7\%).

Conclusions: BZD/SHDs were frequently used by hospitalized patients in Nova Scotia. Areas identified for quality improvement included decreasing the use in older adults, preventing inappropriate initiation of BZD/SHDs in hospital, and improving the documentation of indications for BZD/SHD use.

Keywords: benzodiazepines, sedative-hypnotic drugs, hospital, drug safety, geriatrics, sleep 


\section{Voriconazole Prophylaxis in Leukemic Patients: A Retrospective Single Centre Study Pharmacotherapy Best Practices Award, sponsored by Pfizer Canada ULC}

Bui $V^{1}$, Walker $S A N^{1,2,3,4^{*}}$, Elligsen $M^{1}$, Vyas $A^{1}$, Kiss $A^{5}$, Palmay $L^{1}$ ${ }^{1}$ Department of Pharmacy, Sunnybrook Health Sciences Centre, Toronto, ON ${ }^{2}$ Leslie L Dan Faculty of Pharmacy, University of Toronto, Toronto, $O N$ ${ }^{3}$ Division of Infectious Diseases, Sunnybrook Health Sciences Centre, Toronto, $O N$

${ }^{4}$ Sunnybrook Research Institute, Sunnybrook Health Sciences Centre, Toronto, $O N$

${ }^{5}$ ICES, Toronto, ON

* Senior author, now retired (affiliations at time of study completion)

Background: Invasive fungal infections (IFIs) commonly occur in acute myeloid and lymphoblastic leukemia (AML and ALL) patients receiving chemotherapy. In these patients, posaconazole prophylaxis is recommended; however, voriconazole may be a less costly alternative. The objective of this study was to evaluate the efficacy and safety of voriconazole prophylaxis in acute leukemia patients.

Methods: A retrospective chart review of inpatients at Sunnybrook Health Sciences Centre between 2005 and 2017 was completed. Hospitalized adult AML and ALL patients who received voriconazole prophylaxis (cases) were compared to patients who received fluconazole or no prophylaxis during chemotherapy (controls). Statistical analyses comparing baseline characteristics, safety, and efficacy outcomes between the study cohorts were completed; and a generalized estimating equation (GEE) was used to balance for any observed baseline differences and / or the effect of repeat patient admissions. A posaconazole literature-based weighted mean risk was compared to the voriconazole risk of IFI identified in this study with patients who had similar characteristics, including: age and primary diagnosis.

Results: Of 490 AML or ALL patients, 83 controls and 92 cases were eligible. Case patients received an average of $24.4 \pm 10.8$ days of voriconazole prophylaxis. The GEE confirmed incidence of proven or probable IFIs with voriconazole was 3.3\% (3/92) versus 7.2\% (6/83) in the control cohort (GEE OR 0.43; 95\% CI -2.3-0.6; p>0.05) and was comparable to the literature reported weighted incidence of IFI with posaconazole $(2.4 \pm 2.1 \%$; $95 \%$ CI $1.3-3.4 \%$; p $>0.05)$. Voriconazole was well tolerated by patients $(91 \% ; 84 / 91 ; 7$ discontinued due to asymptomatic elevated liver function tests).

Conclusions: Voriconazole prophylaxis was found to be safe, effective, and comparable to literature based efficacy data for risk of IFI with posaconazole antifungal prophylaxis in patients with acute leukemia undergoing chemotherapy; and could represent a significant cost advantage.

\section{Retrospective, Multicentre Matched Cohort Study Comparing Safety and Efficacy Outcomes of Intermittent Infusion and Continuous Infusion Vancomycin}

Safe Medication Practices Award, sponsored by HealthPRO Procurement Services Inc.

Ma $N^{1,2}$, Walker $S A N^{1,2,3,4^{*}}$, Elligsen $M^{1}$, Palmay $L^{1}, H o G^{5}$,

Leis JA ${ }^{3,4,6}$, Bansal V', Powis J,6

${ }^{1}$ Department of Pharmacy—Bayview Campus, Sunnybrook Health Sciences Centre, Toronto, ON

${ }^{2}$ Leslie L Dan Faculty of Pharmacy, University of Toronto, Toronto, ON

${ }^{3}$ Division of Infectious Diseases, Sunnybrook Health Sciences Centre, Toronto, ON

${ }^{4}$ Sunnybrook Research Institute, Sunnybrook Health Sciences Centre, Toronto, $O N$

${ }^{5}$ Michael Garron Hospital, Toronto, ON

${ }^{6}$ Faculty of Medicine, University of Toronto, Toronto, $O N$

${ }^{7}$ Sunnybrook Health Sciences Centre-Holland Orthopedic and Arthritic Campus, Toronto, ON

* Senior author, now retired (affiliations at time of study completion)

Background: Patients with good renal function receiving intermittent infusion vancomycin (IIV) may require total daily doses $\geq 4 \mathrm{~g}$ to achieve trough concentrations of $15-20 \mathrm{mg} / \mathrm{L}$, increasing the risk of vancomycin associated nephrotoxicity (VAN). Continuous infusion vancomycin (CIV) may enable attainment of target concentrations with a lower daily vancomycin dose, potentially reducing the risk of VAN.

Objectives: The primary objective was to compare VAN risk (serum creatinine $[\mathrm{sCr}]$ increase $\geq 50 \%$ from baseline) and renal damage ( $\mathrm{sCr}$ increase $\geq 100 \%$ from baseline) in patients receiving IIV versus CIV. The secondary objective was to compare clinical cure between IIV and CIV in patients with vancomycin trough or steady state concentrations of $\geq 12 \mathrm{mg} / \mathrm{L}$, respectively.

Methods: Retrospective chart reviews for eligible inpatients at Sunnybrook Health Sciences Centre; Holland Orthopaedic and Arthritic Centre; St. John's Rehab and Michael Garron Hospital between January 1, 2010 and December 31, 2016 were completed. Adult inpatients who received $\geq 48$ hours of vancomycin with $\geq 1$ steady state vancomycin concentration were eligible. Baseline patient characteristics, safety and efficacy outcomes for the IIV and CIV cohorts were compared using appropriate statistical tests (Fisher's exact, Student's t-test, or MannWhitney), with significance defined as $\mathrm{P}<0.05$. A generalized estimating equation model was used to identify independent predictors of VAN.

Results: Of 2136 patients who received vancomycin during the study period, 146 CIV patients were eligible and matched to 146 IIV patients. CIV was found to have a lower odds of developing nephrotoxic risk (odds ratio $[\mathrm{OR}] 0.42,95 \%$ confidence interval $[\mathrm{CI}] 0.21-0.98, \mathrm{p}=0.025)$ and renal injury (OR $0.19,95 \%$ CI $0.05-0.59, \mathrm{p}=0.004)$. There was no difference in clinical cure between IIV (62/67 [93\%]) and CIV patients (58/62 [94\%]; P>0.99).

Conclusion: CIV patients were less likely to experience nephrotoxic risk and renal damage. The results indicate there is no difference in clinical cure between patients who received IIV versus CIV.

Keywords: vancomycin, intermittent infusion, continuous infusion, safety, efficacy 


\section{CSHP NATIONAL AWARDS PROGRAM / PROGRAMME NATIONAL DES PRIX 2019 DE LA SCPH}

\section{Development of a Vancomycin Competency-Based Training and Assessment Program (VC-TAP) Teaching, Learning and Education Award, sponsored by Pfizer Canada ULC}

Rieger $M^{1}$, Evans $B^{1,2}$, Kosar $J^{1}$

${ }^{1}$ Pharmacy Services, Saskatchewan Health Authority, Saskatoon, SK

${ }^{2}$ College of Pharmacy and Nutrition, University of Saskatchewan,

Saskatoon, $S K$

Background: Competency based education focuses on the knowledge, skills and attitudes required to be competent in an area. Currently, hospital pharmacists in Saskatoon receive training for vancomycin; however, competency is not measured.

Objectives: 1) Obtain Saskatoon pharmacists' views on the current training module, existing knowledge gaps, and components of a competency-based training program, 2) define competencies for managing patients on vancomycin, 3) create a vancomycin pharmacist competency rubric and assessment tool, 4) develop vancomycin education modules, and 5) survey pharmacists for feedback on the education modules and future program development.

Methods: Focus groups were conducted to identify what an ideal vancomycin training program would constitute. Competencies and a rubric with accompanying formative feedback form were created using existing standards, focus group discussions, and local expert opinions. Pharmacists were invited to listen to education modules, adapted from the previous vancomycin training PowerPoint $\AA$, and then provide feedback on strengths, perceived gaps and future program development.

Results: Several competencies were defined for pharmacists monitoring patients on vancomycin therapy. The rubric and formative feedback form used the Dreyfus Skill Acquisition rating scale to evaluate pharmacists during initial and continued management of vancomycin. Four vancomycin e-learning modules were created discussing 1) pharmacology and adverse effects, 2) nomograms, dosing, and administration, 3) therapeutic drug monitoring, and 4) dosing in special patient populations. Overall, $29.7 \%$ of hospital pharmacists in Saskatoon completed the education modules and survey. The survey indicated the modules were long but had good content and were well presented.

Conclusions: The rubric, formative feedback form, and education modules have been developed for vancomycin competency-based training and assessment program (VC-TAP). Further study will be required to determine the program's impact on pharmacist competency when dosing and monitoring patients on vancomycin.

Keywords: Competency-based education, pharmacist, vancomycin 